\title{
Estudo das manifestações patológicas em reservatórios de concreto armado por meio de análises estatísticas
}

\author{
D. C. Gomes ${ }^{1 *}$, R. de A. Garcia Sobrinho ${ }^{2}$, R. V. Ribeiro ${ }^{3,}$ Lima M. K. G. de ${ }^{1,}$ \\ *Contato do Autor: eng.demostenes@gmail.com \\ ${ }^{1}$ Faculdade de Engenharia Civil, Universidade de Uberaba, Uberlândia, Brasil \\ ${ }^{2}$ Instituto Master de Ensino Presidente Antônio Carlos, Araguari, Brasil \\ ${ }^{3}$ Departamento de Engenharia Mecânica, Universidade Federal de Uberlândia, Uberlândia Brasil
}

\begin{abstract}
RESUMO
O presente trabalho mostra a análise de patologias em reservatórios de concreto, considerando análise estatística de variância e testes não paramétricos de comparação de grupos independentes. Foram analisadas diversas variáveis que representam condições de execução, concentrações de cloro residual as quais as paredes dos reservatórios estão expostas, idade do reservatório, tipo de reservatório, análise do cobrimento e dos tipos de tratamentos de junta de concretagem. Tal análise buscou estabelecer um método estatístico para explicar a variação da quantidade de patologias em função das variáveis independentes analisadas. Foi possível verificar que o modelo proposto consegue mostrar quais variáveis são realmente significativas na formação de manifestações patológicas.
\end{abstract}

Palavras-chave: Manifestações patológicas, reservatório de concreto, análise estatística

\begin{abstract}
The present work shows the analysis of pathologies in concrete reservoirs, considering statistical analysis of variance and non-parametric tests for comparing independent groups. Several variables were analyzed that represent execution conditions, concentrations of residual chlorine to which the walls of the reservoirs are exposed, age of the reservoir, type of reservoir, analysis of the coverage and types of concreting joint treatments. Such analysis sought to establish a statistical method to explain the variation in the number of pathologies as a function of the independent variables analyzed. It was possible to verify that the proposed model can show which variables are really significant in the formation of pathological manifestations.
\end{abstract}

Keywords: Pathological manifestation; concrete reservoir; statistical analysis

\section{EL RESUMEN}

El presente trabajo muestra el análisis de patologías en reservorios concretos, considerando análisis estadístico de varianza y pruebas no paramétricas para comparar grupos independientes. Se 
analizaron varias variables que representan condiciones de ejecución, concentraciones de cloro residual a las que están expuestas las paredes de los embalses, edad del embalse, tipo de embalse, análisis de la cobertura y tipos de tratamientos de juntas de hormigonado. Dicho análisis buscó establecer un método estadístico para explicar la variación en el número de patologías en función de las variables independientes analizadas. Se pudo comprobar que el modelo propuesto puede mostrar qué variables son realmente significativas en la formación de manifestaciones patológicas.

Palabras clave: Manifestaciones patológicas, reservorio de hormigón, análisis estadístico.

\section{INTRODUÇÃO}

A vida útil e o desempenho das estruturas estão relacionados com as especificações previstas em projetos e com os métodos construtivos adotados. Verifica-se que os requisitos propostos no sistema normativo nacional, especialmente na ABNT NBR 6118:2014 e ABNT NBR 14931:2014, trazem diversas recomendações com o intuito de mitigar problemas advindos de falhas em projetos e erros em execuções.

Nos mesmos sistemas normativos, observa-se a fixação de um prazo de vida útil ideal para estruturas de concreto armado. Na ABNT NBR 15575-1:2013, tem-se que o tempo de vida útil para estruturas é de no mínimo 50 anos, com valor intermediário de 63 anos e valor máximo de 75 anos.

Para se obter o desempenho adequado das estruturas, não se deve apenas considerar os critérios para elaboração de projetos e execução de estruturas. Além deles, tem que se cumprir um plano de manutenção, de acordo com o preconizado na ABNT NBR 5674:2012, que também é essencial para garantir a vida útil dos elementos estruturais.

Apesar das várias recomendações normativas, dos critérios prefixados e dos estudos sobre o tema, é possível observar que diversas estruturas apresentam manifestações patológicas afetando diretamente seu desempenho. Dentre estas estruturas tem-se os reservatórios executados em concreto armado com a finalidade de distribuição de água potável. Estes tipos de estruturas são destinadas ao armazenamento de grandes volumes de água tratada em centros urbanos. O sistema de abastecimento de água depende diretamente do desempenho desses reservatórios, portanto, falhas em seu funcionamento acarretam problemas de desabastecimento, gastos excessivos de reparo e desperdício de água tratada.

Considerando a relevância dos reservatórios e sua necessidade de operação sem a paralisação do abastecimento de água, torna-se importante o estudo das manifestações patológicas deste sistema construtivo. Com o intuito de traçar diagnósticos mais confiáveis e precisos para avaliação e análise de problemas em estruturas de concreto, tem-se como alternativa a análise das anomalias por intermédio de métodos que envolvam análises estatísticas. Dessa forma, pode-se elaborar procedimentos e métodos matemáticos para análise das patologias e apresentar resultados com maior confiabilidade.

Para Mehta e Monteiro (2014), a modernização e o avanço das pesquisas e dos métodos de dosagem possibilitaram a criação de concretos mais duráveis e com melhor desempenho, contudo, o conceito de durabilidade não é absoluto, pois limita-se à vida útil dos elementos estruturais em análise. Com o intuito de contribuir com a melhoria de reservatórios de concreto e proporcionar condições para que estes atinjam satisfatoriamente sua vida útil, diversos pesquisadores investigaram manifestações patológicas e analisaram terapias construtivas nestas estruturas, conforme Tabela 1. De acordo com Souza e Ripper (1998), Sollero e Bolorino (2016) e Lima (2011) apud Gomes (2020) a infiltração de água em reservatórios é um fator preponderante para o surgimento de anomalias. Segundo os autores, ainda existem diversas anomalias estruturais que podem ocorrer 
neste tipo de estrutura. Dentre elas, destacam-se: umidade, fissuras e trincas, carbonatação, corrosão de armadura, eflorescência/lixiviação e desagregação/desplacamento.

Tabela 1 - Panorama de pesquisas em patologias em reservatórios de concreto no Brasil

\begin{tabular}{|c|c|}
\hline Autores & Pesquisa sobre reservatórios de concreto \\
\hline Olivan et al. (2010) & $\begin{array}{c}\text { Vistoria de 197 reservatórios e as suas } \\
\text { anomalias estruturais no Estado de São } \\
\text { Paulo. }\end{array}$ \\
\hline Pereira (2010) & $\begin{array}{c}\text { Vistoria sobre anomalias estruturais em 27 } \\
\text { reservatórios em Portugal. }\end{array}$ \\
\hline Tavassoli e Afshar (2017) & $\begin{array}{c}\text { Estudo de deterioração de reservatórios de } \\
\text { concreto na cidade de Bandar Abbas, no Irã. }\end{array}$ \\
\hline Rachid e Londero (2013) & $\begin{array}{c}\text { Análise de patologias em 10 reservatórios de } \\
\text { distribuição no Paraná }\end{array}$ \\
\hline Corrêa e Girardi (2018) & $\begin{array}{c}\text { analisaram 19 reservatórios na cidade de } \\
\text { Porto Alegre }\end{array}$ \\
\hline
\end{tabular}

Além desses fatores, verificasse que o teor de cloro na água tratada também pode influenciar na vida útil dos reservatórios. De acordo com a Portaria MS 888/2021, toda a água tratada destinada ao consumo humano deve manter valores mínimos de concentração de cloro. Para que se atenda a este requisito, as estruturas de concreto que armazenam água tratada são expostas constantemente a este agente químico. Segundo Pereira (2010), o cloro age diretamente como causador de deterioração do concreto armado e corrosão de armaduras, diminuindo significativamente sua vida útil.

Portanto, tem-se como objetivo apresentar procedimentos estatísticos para análise de manifestações patológicas, levando-se em conta a ocorrência de anomalias como variáveis dependentes e propor procedimento de análise estatística, considerando-se os fatores que contribuem para a formação de patologias em reservatórios de concreto utilizados para distribuição de água potável.

\section{PROCEDIMIENTO}

A pesquisa foi realizada na cidade de Uberlândia, localizada no estado de Minas Gerais no Brasil. Foram investigados 10 reservatórios que correspondem ao total de reservatórios de concreto de Uberlândia. A produção do trabalho pautou-se na implementação dos seguintes procedimentos:

- Coleta de dados laboratoriais: Coletou-se dados laboratoriais com os valores de teor de Cloro livre nos reservatórios (localizados no interior das Estações de Tratamento de Água) e nas redes de distribuição (interligadas aos reservatórios de distribuição).

- Visitas técnicas a todos os reservatórios: Foram elaboradas visitas técnicas com o objetivo de registrar, analisar e catalogar as patologias de cada estrutura e coletar dados sobre as edificações.

- Tratamento estatístico dos dados da amostra: Após a coleta dos dados procedeu-se ao trabalho estatístico com os dados, definindo-se os tipos de variáveis independentes, os níveis de cada variável, os valores dos parâmetros e os intervalos de confiança. Após o tratamento estatístico dos dados, foi possível fazer organização dos dados, de maneira a possibilitar a aplicação de critérios estatísticos de análise.

- Análise estatística dos dados: Elaborou-se análise de variância e análise das variáveis independentes em relação à homogeneidade da variância, teste de normalidade e teste do 
p-valor. Com as demais variáveis independentes que não atenderam aos requisitos necessários para análise de variância, efetuou-se os testes estatísticos não paramétricos.

- Discussão dos resultados: Com os resultados obtidos, procedeu-se à discussão dos valores, apresentação da correlação entre as variáveis e o surgimento das patologias analisadas na amostra.

Com a coleta dos dados laboratoriais e as visitas realizadas, levantou-se as variáveis que foram utilizadas paras as análises do estudo. Elas são: A incidência de manifestações patológicas; o teor de cloro residual livre; o tipo do reservatório; a idade do reservatório; a especificação técnica da junta de concretagem; o cobrimento da armadura e a impermeabilização da parte superior.

Para cada variável qualitativa foram atribuídos valores para a modelagem estatística. A incidência de manifestações patológicas foi definida como a variável dependente, sendo o ponto de investigação do estudo. Em relação aos tipos de manifestações encontrados, foram detectadas 6 tipologias específicas:

- Carbonatação - Ca;

- Corrosão de armadura - Co;

- Desagregação/ Desplacamento - Dd;

- Eflorescência/ lixiviação - Ef;

- Fissuras e trincas - Ft;

- Umidade - Um.

Para as investigações, foi considerado a quantidade de incidência de tipos diferentes de anomalias em cada reservatório, podendo variar de 1 a 6 , de acordo comas manifestações levantadas acima. Essa variável foi definida como quantitativa.

Em relação ao teor de cloro, obteve-se média populacional $(\mu)$ do teor de cloro residual livre na água a partir dos dados laboratoriais do Departamento de Água Esgoto ano de 2017 até o ano de 2019, nas redes de distribuição e nas estações de tratamento de água. Considerou-se, para a modelagem estatística, essa variável como sendo do tipo qualitativa dicotômica, com média de 95\% de confiança que assume os valores 1 ou 2, de acordo com a descrição:

- $\quad(0,7<\mu<0,8)$ : Concentração de cloro residual livre, em mg/L, nas redes de distribuição assume o valor 1

- $\quad(1,04<\mu<1,11)$ : Concentração de cloro residual livre, em mg/L, nas estações de tratamento de água - assume o valor 2

Os reservatórios analisados foram enquadrados em dois tipos, definindo-se como uma variável estatística qualitativa e dicotômica da seguinte forma:

- Reservatório de concreto armado semienterrado - assume o valor 1

- Reservatório de concreto armado elevado - assume o valor 2

De acordo com a data do termino da execução de cada obra, definiu-se a idade do reservatório como sendo uma variável qualitativa ordinal, que assume valores 1,2 ou 3 , de acordo com a descrição:

- 5 a 30 anos - assume o valor 1

- 30 a 55 anos - assume o valor 2

- 55 a 80 anos - assume o valor 3

A variável especificação técnica de junta foi considerada como do tipo dicotômica, pois na amostra há ocorrência de apenas dois tipos tais variáveis. Para a coleta dos dados a respeito dessas variáveis, foram analisados projetos dos reservatórios e verificado se em tais documentos tinha especificação técnica das juntas de concretagem ou projeto executivo dessas juntas de concretagem. De acordo com a coleta de dados, definiu-se os seguintes parâmetros para a variável qualitativa:

- Sem especificação técnica construtiva da junta de concretagem - assume o valor de 0

- Com especificação técnica construtiva da junta de concretagem - assume o valor de 1 
O cobrimento da armadura foi levantado por meio dos projetos estruturais e inspeções no local. Durante a pesquisa, observou-se duas espessuras médias de cobrimento das paredes dos reservatórios de concreto. Diante do fato, foi considerado a variável do tipo qualitativa continua que pode assumir os valores de 3 e $4 \mathrm{~cm}$.

A impermeabilização da parte superior do reservatório, definiu-se tal parâmetro de forma dicotômica, que pode assumir os seguintes valores:

- Parte Superior não impermeabilizada - assume o valor de 0

- Parte Superior impermeabilizada - assume o valor de 1

$\mathrm{Na}$ modelagem estatística, utilizou-se os testes de Shapiro Wilk e de Bartlett para verificar a normalidade e homogeneidade na incidência de patologias. As variáveis cobrimento de armadura e especificação técnica de junta de concretagem foram submetidas a análise de variância ANOVA. Já as variáveis concentração de cloro, tipo de reservatório, impermeabilização da parte superior e idade do reservatório não atenderam o pressuposto para execução da análise de variância, sendo assim, realizou-se testes não paramétricos para definir as influências destas na ocorrência de patologias. Para concentração de cloro, tipo de reservatório e impermeabilização da parte superior foi feito o teste de Mann Whitney; para a idade do reservatório, utilizou-se o teste não paramétrico de Kruskall-Wallis.

Com todos os resultados, foram realizadas as discussões e levantadas as contribuições da pesquisa para o ambiente tecnológico e cientifico.

\section{RESULTADOS}

Na Tabela 2 é possível verificar todas as variáveis levantados por reservatório de acordo com os valões atribuídos para a modelagem estatística. É possível observar, qualitativamente, que os reservatórios 1, 2 e 3 são os que apresentam mais tipologias de manifestações patológicas sendo eles: semi-enterrados, com maior quantidade de cloro livre, menor idade, sem especificação de junta técnica, menor cobrimento e sem impermeabilização da parte superior.

A partir da tabela foram realizadas as análises estatísticas para verificar a influência das variáveis na incidência das manifestações patológicas de forma quantitativa.

A variável de incidência de patologias apresentou significância de 1\% nos testes de Sapiro Wilk e Bartlett confirmando, assim, o pressuposto de homogeneidade de suas variâncias. Em relação a aplicação da ANOVA nas variáveis cobrimento de armadura e especificação do tratamento da junta de concretagem, constatou-se que o $p$-valor de ambos e a interação entre os fatores foi significativa, rejeitando-se a hipótese nula de igualdade de médias entre os tratamentos.

A variável de incidência de patologias apresentou significância de $1 \%$ nos testes de Sapiro Wilk e Bartlett confirmando, assim, o pressuposto de homogeneidade de suas variâncias. Em relação a aplicação da ANOVA nas variáveis cobrimento de armadura e especificação do tratamento da junta de concretagem, constatou-se que o $p$-valor de ambos e a interação entre os fatores foi significativa, rejeitando-se a hipótese nula de igualdade de médias entre os tratamentos.

Foi elaborado o gráfico de interação entre os fatores cobrimento de armadura $(\mathrm{cm})$ e especificação técnica do tratamento da junta de concretagem, dado que as duas variáveis independentes apresentaram combinações entre os níveis de cada fator. Por intermédio da análise estatística, comprovou-se que ambos os fatores influenciam de maneira individual nas patologias e que as combinações dos fatores aumentam significativamente a quantidade de patologias nos reservatórios analisados.

Tabela 2 - Variáveis investigadas de acordo com as atribuições para a modelagem estatística 


\begin{tabular}{|c|c|c|c|c|c|c|c|}
\hline Reserv. & $\begin{array}{c}\text { Incid. de } \\
\text { Manifest. } \\
\text { Pat. }\end{array}$ & $\begin{array}{c}\text { Teor } \\
\text { de } \\
\text { cloro } \\
\text { resid. }\end{array}$ & $\begin{array}{c}\text { Tipo } \\
\text { de } \\
\text { reserv. }\end{array}$ & Idade & $\begin{array}{c}\text { Especi. } \\
\text { técnica } \\
\text { de junta de } \\
\text { concret. }\end{array}$ & $\begin{array}{c}\text { Cobri. } \\
\text { de } \\
\text { arma. }\end{array}$ & $\begin{array}{c}\text { Imperme. } \\
\text { da parte } \\
\text { superior }\end{array}$ \\
\hline Reserv. 1 & 6 & 2 & 1 & 1 & 0 & 3 & 0 \\
\hline Reserv. 2 & 5 & 2 & 1 & 1 & 0 & 3 & 0 \\
\hline Reserv. 3 & 5 & 2 & 1 & 1 & 0 & 3 & 0 \\
\hline Reserv. 4 & 3 & 1 & 2 & 3 & 1 & 3 & 1 \\
\hline Reserv. 5 & 3 & 1 & 2 & 3 & 0 & 4 & 1 \\
\hline Reserv. 6 & 3 & 1 & 2 & 2 & 0 & 4 & 1 \\
\hline Reserv. 7 & 2 & 2 & 2 & 2 & 1 & 4 & 0 \\
\hline Reserv. 8 & 2 & 1 & 2 & 2 & 1 & 4 & 1 \\
\hline Reserv. 9 & 2 & 1 & 2 & 3 & 1 & 3 & 1 \\
\hline Reserv. 10 & 2 & 1 & 2 & 2 & 1 & 4 & 1 \\
\hline
\end{tabular}

Observa-se que quando há a combinação de especificação técnica ou elaboração de projeto executivo de juntas de concretagem, aliado com aumento do cobrimento de armadura, a quantidade de patologias diminui de forma significativa. Tal combinação de dados mostra interação direta entre os fatores, indicando que dentro da amostra analisada tais critérios devem ser rigorosamente observados na fase de elaboração de projetos e fiscalização da execução das obras deste tipo de reservatório, conforme mostra a Figura 1.

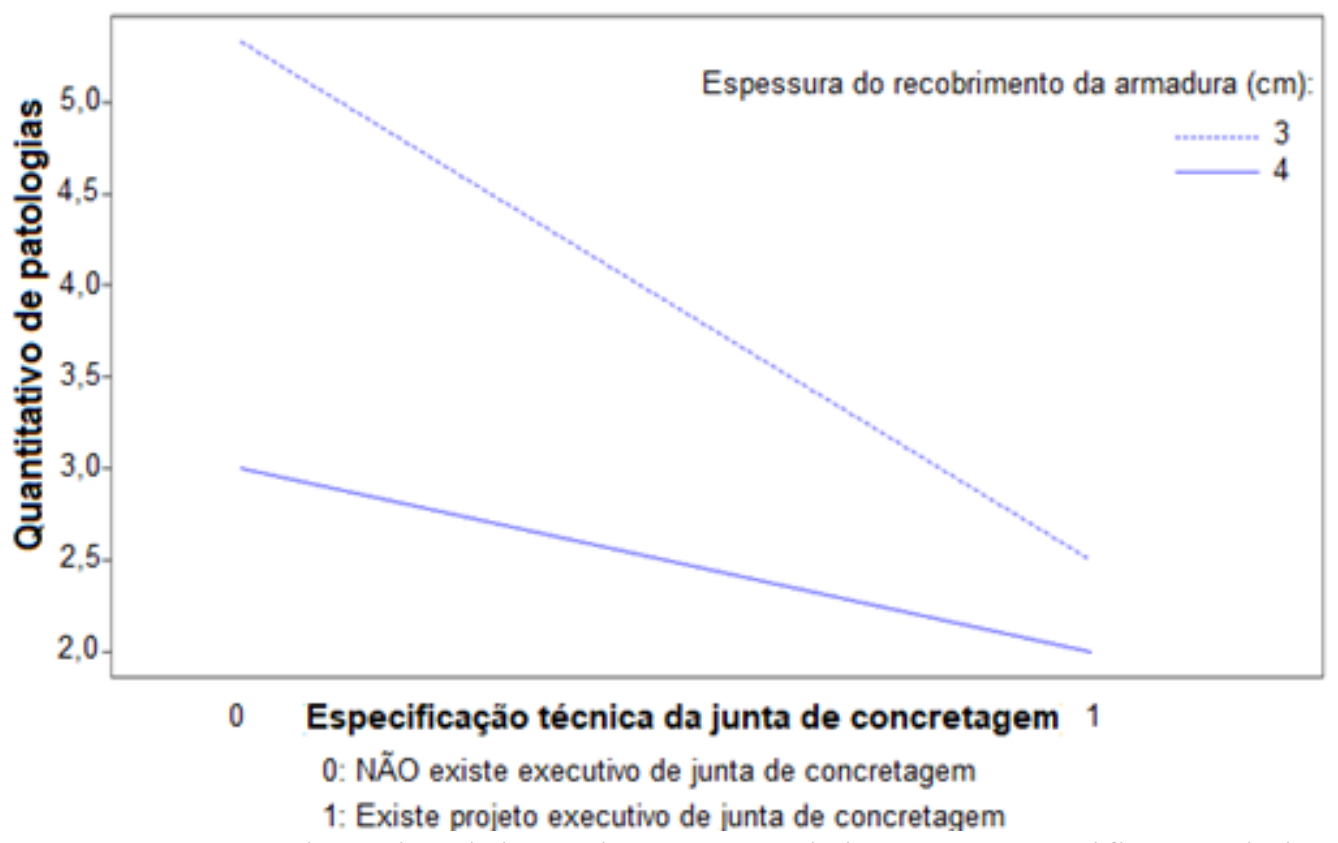

Figura 1. Interação de variável dependente com cobrimento e especificação de junta

Com a análise de variância obteve-se os resultados dos testes e do P-valor que confirmam a analiticamente a hipótese analisada, de acordo com a Tabela 3.

Os testes não paramétricos, utilizados para as variáveis concentração de cloro, tipo de reservatório e impermeabilização da parte superior e idade do reservatório, possibilitam realizar comparação entre grupos independente. Desse modo verificou-se se há diferença entre os grupos e se cada variável influencia a variável dependente, ou seja, quantidade de patologias. Na Tabela 4 constam os resultados de $p$-valor para cada variável. 
Tabela 3. Anova com dois fatores

\begin{tabular}{|c|c|c|c|c|c|}
\hline Fonte de Variação & GL & SQ & QM & Teste F & P-valor \\
\hline Especificação de juntas & 1 & 12,1 & 12,1 & 62,23 & 0,000 \\
\hline Cobrimento & 1 & 4,82 & 4,82 & 24,77 & 0,003 \\
\hline $\begin{array}{c}\text { Especificação de juntas: } \\
\text { cobrimento }\end{array}$ & 1 & 2,02 & 2,02 & 10,37 & 0,018 \\
\hline Resíduo & 6 & 1,17 & 0,19 & & \\
\hline
\end{tabular}

Tabela 4. Valores comparação entre grupos

\begin{tabular}{|c|c|}
\hline Variável & $\boldsymbol{p}$-valor \\
\hline Idade & 0.03 \\
\hline Concentração de cloro & 0.55 \\
\hline Tipo de Reservatório & 0.02 \\
\hline Impermeabilização da parte superior & 0.11 \\
\hline
\end{tabular}

De acordo com os pressupostos teóricos, o grau de confiança do método proposto é $95 \%$, portanto, todas variáveis que apresentam valores maiores que 5\% nos testes não paramétricos, não contribuem significativamente com a variável dependente.

Conclui-se que dentre as variáveis independentes analisadas, apenas as variáveis idade e tipo de reservatório atenderam ao pressuposto de $5 \%$ de significância, ou seja, tais variáveis são significativas e devem ser analisadas no momento da concepção do projeto.

A análise da variável "Tipo de Reservatório" indica que os reservatórios elevados possuem tendência a apresentar ocorrências de patologias inferiores à quantidade de patologias observadas em reservatórios semienterrados, indicando que os projetos e métodos construtivos de reservatórios semienterrados devem ser melhorados em relação aos executados atualmente.

A pesquisa em relação à variável "idade" mostra que de acordo com sua significância e após a realização da comparação múltipla dois a dois, apenas as faixas etárias de idade de 5 a 30 anos apresentaram média de quantidade patologias diferentes, com valores próximos aos limites máximos estudados para amostra, indicando que os projetos e técnicas construtivas atuais não são mais adequados para construção dos reservatórios de concreto, conforme Tabela 5.

Tabela 5. Média de patologias por idade

\begin{tabular}{|c|c|}
\hline Idade & Média de patologias \\
\hline 5 a 30 & 5,33 \\
\hline 30 a 55 & 2,25 \\
\hline 55 a 80 & 2,67 \\
\hline
\end{tabular}

\section{CONCLUSÕES}

Neste trabalho buscou-se propor uma análise estatística de patologias, utilizando-se variáveis qualitativas. Esta metodologia foi adotada, com intuito de elaborar métodos científicos de análises aplicáveis a situações em que se trabalha também com variáveis dicotômicas. Este trabalho pautou- 
se em sugerir método analítico de estruturas, de maneira a proporcionar grau de confiança na otimização das construções e diminuição de patologias em reservatórios de concreto.

Com a revisão da literatura, pôde-se avaliar a confiabilidade da análise estatística elaborada nesta pesquisa, pois em ambos casos fica demonstrado que o cobrimento da armadura e o detalhamento executivo das juntas de concretagem são preponderantes no bom desempenho de reservatórios de distribuição.

A principal contribuição deste trabalho foi estabelecer um método estatístico de análise de patologias. Tal método possibilita que seja feita uma análise científica de tais problemas sem depender exclusivamente da subjetividade. Ressalta-se que o método proposto possibilita fixar critérios científicos de análise em situações estatisticamente adversas, ou seja, com grande número de variáveis independentes, variáveis qualitativas e amostra pequena.

Com o método de modelagem proposto, mesmo sem a concepção de um modelo numérico que pudesse representar todas as situações, foi possível verificar a forma, interação entre as variáveis e as contribuições que cada fator apresenta em relação ao surgimento de patologias em reservatórios. Este método de análise, mesmo com grande quantidade de variáveis independentes qualitativas, revela que pode ser feita análise matemática de patologias por meio de estatística não paramétrica. Mesmo que a área abordada seja carente de mais estudos, pelo conteúdo dos testes, se observa que é possível incorporar técnicas de análise de segurança e risco na metodologia proposta. Destaca-se que a utilização das ferramentas expostas neste trabalho revela que a análise pode ser implementada em conjunto com estudos de métodos probabilísticos de análise de patologias. O conceito proposto nesta pesquisa poderá ser utilizado em trabalhos que se propõem a estudar riscos e explicar patologias estruturais a partir da coleta de dados quantitativos e qualitativos.

\section{REFERÊNCIAS}

ASSOCIAÇÃO BRASILEIRA de NORMAS TÉCNICAS. (2013) NBR 15575-1: Edificações habitacionais - Desempenho, Parte 1: Requisitos gerais. Rio de Janeiro

ASSOCIAÇÃO BRASILEIRA de NORMAS TÉCNICAS. (2014) NBR 14931: Execução de Estruturas de Concreto - Procedimento. Rio de Janeiro

ASSOCIAÇÃO BRASILEIRA DE NORMAS TÉCNICAS. (2012) NBR 5674: Manutenção de edificações - Requisitos para o sistema de gestão de manutenção. Rio de Janeiro.

ASSOCIAÇÃO BRASILEIRA de NORMAS TÉCNICAS. (2014) NBR 6118 (NB 1/2014):

Projeto de Estruturas de Concreto - Procedimento. Rio de Janeiro

BRASIL - MINISTÉRIO DA SAÚDE. Portaria $\mathrm{n}^{\circ} 888$ de 04 de maio de 2021. Dispõe sobre os procedimentos de controle e de vigilância da qualidade da água para consumo humano e seu padrão de potabilidade. Diário Oficial da União, 2021.

CORRÊA, P. R. D.; GIRARDI, R. (2018) Análise da evolução dos parâmetros de dimensionamento de reservatórios em concreto: estudo de caso. "Revista de Engenharia Civil IMED, Passo Fundo", v. 5, n. 1, p. 52-66.

GOMES, D. C. "Análise experimental do desempenho de tensores de fôrmas para reservatórios de concreto." (2020) Dissertação, Universidade Federal de Uberlândia, Uberlândia,

LIMA, M. G. In: ISAIA, G. C. (ed.). (2011) "Ações do Meios Ambiente sobre as Estruturas de Concreto. Concreto: Ciência e tecnologia." 1. ed. São Paulo, IBRACON, v. 1. P. 733- 772.

MEHTA, P. K.; MONTEIRO, P. J. M. (2014) "Concrete - Microstructure, Properties, and Materials $-4^{a}$ Ed. Ed.” MC Graw Hill Education. ISBN: 978-0-07-179787-0. Inglês. p.675.

OLIVAN, L. I.; GREGORE, L.; GARRIDO, R.; TAKI, O.; FERREIRA, T. (2010) Estruturas de concreto para abastecimento de água. Um estudo das incidências das anomalias e causas. In: " $52^{\circ}$ Congresso Brasileiro de Concreto IBRACON”, Fortaleza, CE, Brasil. 
PEREIRA, E. A. (2010) "Patologias em reservatórios de água potável e sua correcção." Dissertação (Mestrado), SEL - Eng. Civil, Lisboa.

RACHID, L. E. F.; LONDERO, C. (2013) Levantamento de patologias em reservatórios de concreto armado no município de Cascavel-PR. "Revista técnico-científica do CREA-PR”, v.1, n. 1.

SOLLERO, M.; BOLORINO, H. (2016) Investigation and diagnosis of a reinforced concrete reservoir with intense crack formation from several sources. "Journal of Building Pathology and Rehabilitation," v. 1, n. 6.

SOUZA, V. C.; RIPPER, T. (1998) "Patologia, recuperação e reforço de estruturas de concreto." $1^{a}$ ed. São Paulo, Pini.

TAVASSOLI, A. H.; AFSHAR, F. Investigating the effect of chlorine ion on corrosion of Bandar Abbas reservoir roof. American Society for Engineering Education, v. 6, n. 2, 2017. 